\title{
El Parlamento del Mercosur: ¿hacia un proceso de integración más democrático? \\ María Victoria Álvarez
}

María Victoria Álvarez es Magister en Integración y Cooperación Internacional - Universidad Nacional de Rosario y Katholieke Univeriteit Leuven, Bélgica.

Mail: mvalvarez@fcpolit.unr.edu.ar

\section{resumen}

La instalación de un Parlamento regional, cuyos miembros serán elegidos a través de sufragio universal directo a partir de 2011, constituye un hito sin precedentes en la historia del bloque. Este trabajo pretende realizar un breve análisis del alcance de la instalación de un órgano de estas características en el Mercosur, especialmente en relación al "déficit democrático" del bloque. Desde un punto de vista político, el Parlamento -que significa una señal de compromiso con la continuidad del proceso de integración- podría ejercer un papel fundamental en la defensa de la democracia y los derechos humanos; así como en la protección de los diversos intereses de la población. Adicionalmente, la elección directa de los parlamentarios podría constituir un ejercicio ciudadano que contribuya a incrementar la representatividad y legitimidad democrática del proceso de integración. Sin embargo, desde una perspectiva jurídica, el Parlamento regional se caracteriza por ser un órgano consultivo, con débiles atribuciones legislativas, presupuestarias y de control. Difícilmente la existencia del Parlamento del Mercosur, tal como ha sido instituido, sea capaz de enmendar el "déficit democrático" del bloque.

\section{palabras claves}

Procesos de integración / Mercosur / Parlamento del Mercosur / déficit democrático

\section{summary}

The creation of a directly elected parliamentary body constitutes a milestone in the history of the bloc. Representatives to the Parliament will be elected by direct, universal and secret ballot in 2011. We analyze the creation of the Mercosur Parliament from both a political and a legal perspective, with emphasis on its effects on the "democratic deficit". From a political point of view, the Mercosur Parliament could play an outstanding role in the defence of democracy and human rights, as well as the protection of interests of Member States' populations. Additionally, direct elections to the Parliament could contribute to enlarge the bloc's democratic legitimacy. From a legal standpoint, however, the regional Parliament's fragile legislative, budgetary and oversight powers seem to hinder any possibility to overcoming the "democratic deficit" of the bloc.

\section{keywords}

Processes of economic integration / Mercosur / Mercosur Parliament / democratic deficit 


\section{Introducción}

Los procesos de integración no se desarrollan en el vacío sino en determinados contextos políticos, sociales y económicos. El desenvolvimiento de las instituciones democráticas internas de los países embarcados en proyectos integracionistas tiene repercusiones en las instituciones comunes.

La consolidación democrática de los países del Mercosur en los últimos años, junto con el compromiso de sus gobiernos respecto al reforzamiento del bloque en el ámbito político, se conjugaron para llevar adelante la creación de un Parlamento regional, elegido por los ciudadanos del Mercosur.

El Parlamento del Mercosur (PM), que entró efectivamente en funciones el 7 de mayo de 2007, está compuesto por parlamentarios designados por sus respectivos congresos nacionales. El nuevo órgano unicameral cuenta con 18 parlamentarios por cada Estado parte del bloque, con un mandato de cuatro años. Además de Argentina, Brasil, Uruguay y Paraguay, participan del Parlamento los representantes de Venezuela, con voz pero sin voto hasta que se complete su proceso de adhesión al bloque.

La novedad principal del PM, indudablemente, reside en el hecho de que sus miembros serán elegidos en forma directa por la ciudadanía a partir de 2011. El Parlamento estará conformado, entonces, por legisladores elegidos por los ciudadanos a través de sufragio universal, convirtiéndose en la única institución del bloque que contará con una representación democrática directa.

Este trabajo pretende realizar un breve análisis del significado que posee la instalación de un órgano de estas características en el Mercosur, teniendo en cuenta que el abordaje de la institucionalización del bloque regional "debe hacerse a partir de las características propias de esta alianza y de su expresión histórica, identificando con realismo necesidades y posibilidades, y no tratando de deducir respuestas institucionales basadas en modelos teóricos o en experiencias de procesos de integración con rasgos diferentes" (Pena y Rozemberg, 2005:14).

A un año de su puesta en marcha efectiva, el Parlamento se constituye en un actor digno de ser analizado, desde dos puntos de vista complementarios. Por un lado, por el significado presente y futuro del Parlamento desde una perspectiva política. En particular, analizaremos en este artículo el papel del PM en la defensa de derechos humanos y en los vínculos con la sociedad civil. Por otro, la justa valoración de un órgano de este tipo resulta incompleta si se excluye el examen jurídico de su naturaleza y atribuciones. Finalmente, incluiremos algunas reflexiones acerca de los efectos que la instalación del Parlamento podría producir respecto al llamado "déficit democrático" del Mercosur.

\section{Perspectiva política}

Tal como afirma Bernal-Meza (2001:39), la institucionalización es la manifestación jurídica de la voluntad política de integrarse, de la decisión política de avanzar en un proceso irreversible de integración. La instalación del Parlamento formaliza la voluntad de los gobiernos del bloque (principalmente de Argentina y Brasil) de afirmar la continuidad del proyecto integracionista. 
La instalación del Parlamento concreta una importante señal política, por parte de los Estados parte, hacia dentro y hacia afuera del Mercosur respecto a la irreversibilidad del proceso de integración. Más aún, implica un intento por dejar de entender al Mercosur solamente como un proyecto económico-comercial para apuntar a convertirse en una empresa de integración más profunda.

Este ha sido el objetivo principal de la política brasileña respecto al Mercosur desde la llegada al gobierno de Luiz Inácio "Lula" da Silva en 2002. El cambio de gobierno implicó un giro respecto a la percepción del proyecto integracionista en el Cono Sur, otorgando mayor importancia a los avances institucionales de tipo político, como la constitución de un parlamento regional elegido mediante sufragio universal (Castro Mendizábal, 2005:13).

La creación de órganos que no habían sido contemplados en los acuerdos originarios, y que surgen con posterioridad, forma parte del "gradualismo" típico del Mercosur, una tendencia a crear organismos a partir de los requerimientos que el proceso va demandando (Pena y Rozemberg, 2005:2). Sin embargo, es materia de controversia si los resultados del proceso de institucionalización del bloque proveen un marco de referencia consistente para el intercambio de capitales, bienes, personas y servicios, tareas imprescindibles en un proceso de integración que aspira a convertirse en un mercado común. En este sentido, se torna vital la necesidad de realizar una reformulación conceptual del Mercosur paralelamente a la creación de instancias institucionales, de manera que la institucionalización sea justificada por la profundización del proceso de integración (Bernal-Meza, 2001:74).

Un proceso de mayor institucionalización, sin embargo, no necesariamente conduce a un incremento de la eficiencia gubernamental, ya que puede suceder que se sustituya la burocracia doméstica por una internacional (Bernal-Meza 2001:40). En particular, existe el riesgo de que el Parlamento regional se convierta en otro órgano burocrático vacío de sentido, que aumente el costo de la estructura institucional sin alcanzar sus cometidos políticos. La introducción de una institución costosa e ineficiente pondría en juego la confianza de las sociedades de los Estados parte en el proyecto integracionista. El crecimiento exponencial de órganos y mecanismos institucionales, además, no lograría disimular las evidentes deficiencias del Mercosur (Czar de Zalduendo, 2004:5).

El Parlamento del Mercosur debería evitar el desprestigio de otras experiencias en América Latina, como el Parlamento Centroamericano, que sufre la amenaza de desaparición bajo acusaciones de ser ineficiente, despilfarrar fondos públicos y proteger a ex-funcionarios corruptos gracias a sus inmunidades. Por el contrario, al otro lado del Atlántico, el Parlamento Europeo ofrece una enseñanza clara en el sentido que la consolidación como institución legislativa es una tarea que requiere tiempo, y sobre todo, voluntad política.

Para que el establecimiento del Parlamento constituya un aporte institucional positivo, y no forme parte de una inconveniente multiplicación de órganos, esta institución debería encarnar la representación política y social de las comunidades de los Estados participantes. En este sentido, las esperanzas se orientaron a la posibilidad de que el Parlamento del Mercosur amplíe la agenda interna del bloque 
y tenga la capacidad de contener, articular y orientar los diversos intereses de las sociedades de los Estados parte que no encuentran cabida en otras instituciones. Inclusive, muchos de los temas conflictivos del bloque podrían ser debatidos en el Parlamento, como foro privilegiado para intentar zanjar las discusiones y alcanzar consensos.

\section{Competencias del PM en el ámbito político}

El Parlamento del Mercosur podría convertirse en un canal privilegiado de conexión con la ciudadanía. En este ámbito, sobresale la función de "organizar reuniones públicas, sobre cuestiones vinculadas al desarrollo del proceso de integración, con entidades de la sociedad civil y los sectores productivos" (art. 4 inciso 9 Protocolo Constitutivo del Parlamento del Mercosur, PCPM, el énfasis es nuestro). Asimismo, el PM puede "recibir, examinar y en su caso canalizar hacia los órganos decisorios, peticiones de cualquier particular de los Estados Partes, sean personas físicas o jurídicas, relacionadas con actos u omisiones de los órganos del Mercosur" (art. 4 inciso 10 PCPM, el énfasis es nuestro). Estas disposiciones resultan sumamente relevantes ya que la calidad del vínculo que el Parlamento establezca con los ciudadanos y la sociedad civil es central para avanzar hacia una cultura política e institucional favorable al proceso de integración.

En este sentido, se trata de un órgano que ha sido concebido para desempeñar un fuerte papel político. El Parlamento tiene importantes funciones referidas a la preservación de la democracia y el respeto de los derechos humanos. Es destacable la obligación del Parlamento de presentar un informe anual sobre la situación de los derechos humanos en la región (art. 4 inciso 3 PCPM, el énfasis es nuestro), el cual podría ser utilizado como instrumento de presión hacia cualquier tentativa de los gobiernos de afrentar esos derechos en su territorio. De particular interés, asimismo, es el deber del Parlamento de velar por la preservación del régimen democrático en los Estados Partes, de conformidad con las normas del Mercosur (art. 4 inciso 2 PCPM, el énfasis es nuestro).

\section{Los logros del PM en el ámbito político}

Desde que entró efectivamente en funciones, en mayo de 2007, el Parlamento del Mercosur ha realizado una tarea por demás discreta en su rol político y de vínculo entre el esquema institucional del Mercosur y la ciudadanía.

Las primeras cinco sesiones ordinarias (de mayo a septiembre de 2007) estuvieron dedicadas a la puesta en marcha y organización interna del Parlamento, que incluyó, entre las cuestiones más importantes, la aprobación del Reglamento interno, la conformación de las comisiones parlamentarias y la organización de los órganos internos del Parlamento.

A partir de la VI sesión ordinaria (octubre 2007) el Parlamento inició su accionar propiamente dicho. La VII sesión ordinaria (noviembre 2007) y la sesión extraordinaria de diciembre de 2007 completaron el primer año de actividad. El resultado de esas reuniones se resume en varias Disposiciones, cuatro Declaraciones y dos Resoluciones. 
De los principales actos jurídicos aprobados, sólo pueden destacarse la Declaración de Apoyo del Parlamento del Mercosur al régimen institucional de Bolivia $^{1}$, de respaldo a las gestiones del Presidente de Venezuela Hugo Chávez para un acuerdo humanitario en Colombia ${ }^{2}$, y la condena al genocidio del pueblo armenio $^{3}$.

Desde el inicio del año 2008, el Parlamento se ha reunido en dos ocasiones: el 31 de marzo (VIII sesión plenaria) y los días 28 y 29 de abril (IX sesión plenaria). Entre los actos aprobados, cabe mencionar una Declaración de aplauso al Grupo de Rio y al Consejo Permanente de la Organización de los Estados Americanos, que condenaron la violación del territorio ecuatoriano por parte de las fuerzas militares de Colombia, durante la operación contra guerrilleros de las Fuerzas Armadas Revolucionarias de Colombia ${ }^{4}$.

Más allá de estas exiguas manifestaciones, algunos temas políticos sensibles dentro del Mercosur no han podido ingresar en el orden del día del Parlamento. Este es el caso de los cortes de puentes internacionales entre Argentina y Uruguay por parte de movimientos ambientalistas argentinos en protesta por la instalación de papeleras sobre el Río Uruguay. La cuestión, de importantes efectos medioambientales y económicos, y que, además, ha generado ásperas rigideces entre los respectivos gobiernos, ha estado ausente del debate parlamentario regional. La insistencia del gobierno argentino sobre la "bilateralidad" del conflicto en contra de cualquier intento de "multilateralización" quedó fielmente reflejada en la constante oposición de los diputados argentinos oficialistas a discutir la cuestión en el recinto pese a constantes pedidos por parte de legisladores uruguayos, quienes consideran a los cortes como una trasgresión al Tratado de Asunción.

La lentitud en la puesta en marcha del Parlamento, la aprobación de declaraciones que no constituyen ningún tipo de aporte a la visibilidad del nuevo órgano, sumado a la falta de debate sobre temas de interés para la ciudadanía de los Estados parte, acrecienta las dudas sobre las condiciones del PM para conducir la representación de las sociedades de los países del bloque.

\section{Perspectiva jurídica}

El análisis en este ámbito gira alrededor de dos cuestiones principales: la supranacionalidad y las competencias del nuevo órgano.

En cuanto a la primera cuestión, funcionarios y especialistas afirman que el establecimiento del parlamento regional representa para el bloque el paso de una estructura institucional exclusivamente intergubernamental a un sistema mixto, al añadir una institución supranacional.

Para que una institución sea supranacional debe cumplir rígidas condiciones -que suponen cesión de soberanía de los Estados-, que no están presentes en el PM. El Parlamento es beneficiario, sin embargo, de algunas disposiciones que lo aproximan a la supranacionalidad.

Uno de estos elementos está expresado en el PCPM: "Los miembros del Parlamento no estarán sujetos a mandato imperativo y actuarán con independencia en el ejercicio de sus funciones" (art. 9). El Parlamento se erige así en un órgano 
independiente y autónomo de los demás órganos del bloque y de los gobiernos de los Estados parte. Adicionalmente, los parlamentarios del Mercosur, al redactar su Reglamento interno, han reforzado esta predisposición. Tal como ocurre en el Parlamento Europeo, el PM podrá organizarse en grupos de acuerdo con sus afinidades políticas (art. 33 Reglamento interno del PM). ${ }^{5}$ La existencia de grupos políticos trans-nacionales es una condición esencial para avanzar en la construcción de una representatividad regional.

El Protocolo del PM ofrece un elemento adicional intrínsecamente supranacional al establecer que el Parlamento adopta sus decisiones y actos por distintas mayorías (mayoría simple, absoluta, especial o calificada, art. 15 PCPM).

Una organización de tipo supranacional tiene capacidad de adoptar decisiones por mayoría en contraposición a los órganos intergubernamentales, que deciden por unanimidad, lo cual otorga un poder de veto a cualquiera de los Estados parte. El mecanismo de mayorías previsto para el PM constituye un rasgo que lo diferencia de su antecesora, la Comisión Parlamentaria Conjunta del Mercosur (CPC), que por su carácter intergubernamental, sólo adoptaba decisiones por consenso.

Sin embargo, el rasgo de supranacionalidad que podría otorgar la forma de tomar decisiones no consigue convertir al Parlamento del Mercosur en un órgano supranacional.

En primer lugar, las decisiones del PM carecen de estatus superior a las normas de los parlamentos nacionales; la normativa del bloque es creada por los órganos decisorios intergubernamentales, y continúa vigente la necesidad de incorporar dicha normativa a los respectivos ordenamientos jurídicos nacionales. Las normas Mercosur carecen de aplicabilidad inmediata y efecto directo, característico de esquemas supranacionales.

En segundo lugar, la relevancia del procedimiento de decisión por mayoría resulta reducida por el hecho de que las competencias legislativas, de control y presupuestarias del Parlamento son sumamente débiles.

\section{Competencias legislativas, de control y presupuestarias del PM}

La existencia de un parlamento regional conlleva ventajas a un proceso de integración por su contribución a la función legislativa. Las competencias del Parlamento en este ámbito son cualitativa y cuantitativamente superiores a las de la CPC. Ello no implica, sin embargo, que el Parlamento sea propiamente un órgano legislativo.

Por un lado, las llamadas atribuciones legislativas del Parlamento del Mercosur no atañen estrictamente la "creación" de normas supranacionales, pese a que la variedad de actos jurídicos que puede generar se ve acrecentada, al poder "emitir declaraciones, recomendaciones e informes sobre cuestiones vinculadas al desarrollo del proceso de integración" (art. 4 inc. 11 PCPM), "proponer proyectos de normas Mercosur al Consejo Mercado Común (CMC)" (art. 4 inc.13) o "elaborar estudios y anteproyectos de normas nacionales" (art. 4 inc. 14).

Por otro lado, se configura un procedimiento que requiere el dictamen del PM sobre proyectos de normas del Mercosur. Los órganos decisorios del bloque tienen 
la obligación de transmitir al Parlamento los proyectos de normas antes de ser aprobados, para que éste emita un dictamen. Si el proyecto de norma es aprobado por el órgano decisorio de conformidad con los términos del dictamen del Parlamento, la norma debe ser considerada por los poderes legislativos nacionales dentro de los 180 días siguientes a través de un procedimiento preferencial. Sin embargo, los órganos decisorios del Mercosur no están obligados a aprobar la norma según los términos del dictamen del Parlamento sino que sólo tienen la obligación de solicitarlo (art. 4 inc. 12 PCPM). Pese a ser un avance, este procedimiento no significa la incorporación de una competencia propiamente de creación de legislación.

En definitiva, el Parlamento carece de poderes que le permitan exceder su carácter de órgano básicamente consultivo y asesor. La limitación de sus competencias legislativas es especialmente grave, y crea dudas acerca de la capacidad del Parlamento para avanzar en sus objetivos de armonización de las legislaciones nacionales e incorporación de la normativa del Mercosur a los ordenamientos jurídicos internos, designios fundamentales para acrecentar la seguridad jurídica del bloque.

En cuanto a las competencias de control, el Parlamento del Mercosur supone un adelanto respecto a la CPC. Mientras que ésta podía "solicitar a los órganos institucionales del Mercosur informaciones respecto a la evolución del proceso de integración" (art. 3 inc. c Reglamento interno CPC), el Parlamento puede efectuar pedidos de informes u opiniones por escrito a los órganos decisorios y consultivos del Mercosur sobre cuestiones vinculadas al desarrollo del proceso de integración que deben ser respondidos en un plazo máximo de 180 días (art. 4 inc. 4 PCPM).

El PM, entonces, puede solicitar informes y este pedido debe ser respondido en menos de seis meses. Si bien este plazo es relativamente prolongado, representa una mejora respecto a la CPC, que no preveía ningún lapso de tiempo para que su pedido fuera satisfecho. Adicionalmente, el PM recibe de la Presidencia Pro Tempore del Mercosur un programa de trabajo al inicio de cada semestre, y un informe final sobre las actividades realizadas (art. 4 incisos 6 y 7). Sin embargo, el PM no tiene ninguna potestad sobre el nombramiento o remoción de los miembros de los órganos decisorios intergubernamentales. Los frágiles mecanismos de control institucional del Mercosur no son comparables con el control político instaurado en otros procesos de integración.

Por último, el Parlamento del Mercosur carece prácticamente de atribuciones presupuestarias, ya que sólo es notificado sobre estas cuestiones al recibir, dentro del primer semestre de cada año, un informe sobre la ejecución del presupuesto de la Secretaría Administrativa del Mercosur del año anterior. El presupuesto de la Secretaría en 2008 representa algo más de un millón de dólares.

\section{Los logros del PM en materia legislativa y de control}

Hasta la fecha, el Parlamento del Mercosur se ha limitado a aprobar un puñado de Declaraciones, Resoluciones y Recomendaciones. El órgano parlamentario regional no ha aprovechado las atribuciones que posee (y que no contaba su an- 
tecesora, la CPC) en cuanto a la posibilidad de aprobar anteproyectos de normas -que son las proposiciones orientadas a la armonización de las legislaciones de los Estados Parte, dirigidos a los parlamentos nacionales para su eventual consideración-; y los proyectos de normas del Mercosur, que constituyen las proposiciones normativas presentadas para la consideración del CMC.

El Parlamento tampoco ha tenido la ocasión de llevar adelante el denominado "procedimiento de dictamen consultivo" ya que el CMC todavía no ha enviado proyectos de normas - que requieren aprobación legislativa en uno o más Estados Parte- para que el Parlamento emita su opinión antes de su aprobación.

En cuanto a las tareas de control, el Parlamento, en cumplimiento del PCPM, se limitó a recibir el programa de trabajo al inicio de la Presidencia Pro Tempore de Paraguay para el primer semestre de 2007 y de Uruguay para el segundo semestre de 2007. Asimismo, en la IX Sesión Plenaria recibió del Canciller argentino el informe de la Presidencia Pro Tempore para el primer semestre de 2008.

\section{El debate sobre el "déficit democrático"}

El Parlamento del Mercosur dista de ser un órgano legislativo en tanto el poder reglamentario se mantiene en manos de los órganos decisorios, y sólo se instala un procedimiento que requiere el dictamen del PM aunque no es vinculante. Igualmente, sus potestades de control y presupuestarias son prácticamente inexistentes. El Parlamento se asemeja a un órgano con funciones netamente consultivas sin protagonismo en los procesos de decisión del bloque. Con todo, supone un avance con relación a la CPC. Entonces, ¿se ha conseguido subsanar la falta de "democratización" en el Mercosur con la creación del Parlamento?

Recordemos que el problema del llamado "déficit democrático" es causado, según los especialistas, por la falta de mayor participación del poder legislativo en el proceso de toma de decisiones, esto es, la ausencia de mecanismos propios de control y de creación de normas (Vázquez, 2002:169).

Explicar el "déficit democrático" que se registra en el Mercosur en términos de falta de efectiva intervención del órgano legislativo en el proceso decisorio implica, consiguientemente, que la solución se remitiría a la constitución de un parlamento regional, de características supranacionales, con amplios poderes, que reduzca la hegemonía de los ejecutivos en el bloque. Entendido el déficit en estos términos, difícilmente la existencia del Parlamento del Mercosur, tal como ha sido instituido, sea capaz de enmendarlo.

No obstante, la conveniencia de crear el Parlamento del Mercosur podría basarse en el empeño de dotar de mayor representatividad al proceso de integración, teniendo en cuenta que los parlamentos disfrutan de una función de representación. Según el PCPM, los parlamentarios representan a los pueblos del Mercosur (art. 2). La elección de los miembros del Parlamento por sufragio universal directo se constituiría, así, como un medio para la promoción de la participación ciudadana en el proceso de integración y el aumento de la legitimidad democrática. Asimismo muchos creen que el PM puede coadyuvar a la construcción de un demos e identidad "mercosureñas". 
Estas consideraciones están imbuidas de enfoques que aseveran que las instituciones poseen un valor "constitutivo" y, por lo tanto, pueden contribuir a robustecer el propio proceso de integración regional. A la luz de esta visión, el PM podría apuntar a la construcción de una nueva auto-percepción y de una identidad en relación con el espacio regional (Colacrai, 2005:394).

Sin embargo, es apresurada cualquier conclusión sobre la posibilidad que tengan las elecciones al Parlamento de construir una conciencia de identidad regional, y otorgar mayor legitimidad al proceso de integración. La experiencia en otras partes del mundo indica claramente que la construcción de una identidad regional es una cuestión cultural y no se resuelve a fuerza de tratados o procesos electorales (CERA, 2006:19). En el caso de la Unión Europea, por ejemplo, las elecciones al Parlamento Europeo aún no han logrado crear una verdadera "identidad comunitaria". La continua merma en la concurrencia de los votantes -sumado al hecho de que las elecciones europeas resultan ser referendos sobre el desempeño de los respectivos gobiernos nacionales- son manifestaciones de las persistentes dificultades para alcanzar ese objetivo.

Tal como ha sido concebido, el Parlamento del Mercosur no resuelve por sí solo el importante "déficit democrático" que caracteriza al bloque (Geneyro y Vázquez, 2006:14). Adicionalmente, la posibilidad de avanzar hacia una cultura política e institucional favorable a un proceso de integración cualitativamente diferente no deja de estar condicionada por la situación política que se registre en los países miembros, caracterizada en los últimos años por sucesivas crisis de representatividad y legitimidad democrática (CERA, 2006:14).

\section{Consideraciones finales}

Son diversas las dudas sobre el futuro del Parlamento del Mercosur. Una nueva institución puede implicar formidables costos financieros y políticos si el acrecentamiento de la burocracia no se traduce en respuestas y mayor representatividad para los ciudadanos. La falta de competencias relevantes y el consecuente papel relegado en el proceso de toma de decisiones hacen del Parlamento un órgano jurídicamente irrelevante en el esquema institucional. Por consiguiente, es incierta su contribución en el proceso de internalización de normas y la seguridad jurídica del bloque.

Sin embargo, los beneficios de contar con un órgano parlamentario democrático deberían ser superiores a las desventajas. Desde el punto de vista político, el Parlamento - cuya cristalización significa una señal de compromiso con la continuidad del proceso de integración- puede ejercer un papel fundamental en la defensa de la democracia y los derechos humanos; así como en la protección de los diversos intereses de la población. Adicionalmente, la elección directa de los parlamentarios a partir de 2011 podría constituir un relevante ejercicio ciudadano que contribuya a incrementar la representatividad y legitimidad democrática del proceso de integración.

El logro de este objetivo se cumplirá en la medida en que el Parlamento consiga superar los condicionamientos jurídicos que lo debilitan, maximizar su papel po- 
lítico, y convertirse en un verdadero órgano representativo de los ciudadanos del bloque y en un auténtico legislador de la integración. Difícilmente el proceso de integración logre un carácter democrático si el cuerpo legislativo no es más que un órgano consultivo y no tiene verdadera y efectiva intervención en los procesos de decisión relevantes para la ciudadanía.

El desafío es lograr articular la idea política de un Mercosur democrático y representativo con una institución parlamentaria ambiciosa y eficaz.

\section{Referencias}

1. MERCOSUR/PM/DECL, $05 / 2007$.

2. MERCOSUR/PM/DECL, 03/2007.

3. MERCOSUR/PM/DECL, 04/2007.

4. MERCOSUR/PM/DECL, 03/2008. La declaración incluye la sugerencia de que América del Sur continúe siendo una región en que "predominen la cooperación y la solución negociada de los conflictos, con vistas a su integración en los marcos de democracias prósperas, estables y socialmente justas".

5. Actualmente, el Parlamento del Mercosur cuenta con un grupo político constituido: el Partido Nacional.

\section{Bibliografía}

R. BERNAL-MEZA (2001), "Institucionalización del Mercosur", en D. BENECKE y A. LOSCHKY (editores), Mercosur: Desafio político, Buenos Aires, CIEDLA/Fundación Konrad Adenauer.

CÁMARA DE EXPORTADORES DE LA REPÚBLICA ARGENTINA (CERA) (2006), Parlamento del Mercosur: ¿la voz de los ciudadanos en la integración?, Buenos Aires, Disponible en http://www. cera.org.ar/new-site/documentos/es/139/0.93860000\%201193181416/pdf/PARLAMENTO_DEL_ MERCOSUR.pdf.

M. S. CASTRO MENDIZÁBAL (2005), "La política exterior de Brasil durante el primer año de presidencia de Lula: un marco para el análisis futuro", Ponencia presentada en el XI Encuentro de Estudiantes y Graduados de Relaciones Internacionales del Cono Sur - CONOSUR "El Mandato histórico de la Integración", Montevideo, 6-9 de octubre.

M. COLACRAI (2005), "La marcha de la integración en América Latina. El rol de las ideas, instituciones y políticas en el Mercosur", en A. BORÓN y G. LECHINI, Política y movimientos sociales en un mundo hegemónico. Lecciones desde África, Asia y América Latina, Buenos Aires, CLACSO.

S. CZAR DE ZALDUENDO (2004), "La institucionalización del Mercosur: ¿Ouro Preto II?”, en Puente@Europa, Año 2, No 8.

P. DA MOTTA VEIGA (2005), “A política comercial do Governo Lula: continuidade e inflexão”, Brief \#19, Latin American Trade Network (LATN) - Fundaçao Centro de Estudos do Comércio Exterior, Buenos Aires - Rio de Janeiro.

A. DREYZIN DE KLOR (2004), "La necesidad de un Parlamento para el Mercosur", en Fundación Konrad Adenauer y Comisión Parlamentaria Conjunta del Mercosur (editores), Hacia el Parlamento del Mercosur, Montevideo.

R. GENEYRO y A. MUSTAPIC (2000), "Fortalecimiento de la dimensión parlamentaria e institucional del proceso de integración. Pautas para su futura evolución", en G. CAETANO y R. PERINA (Coord.), Parlamentos e instituciones en el Mercosur. Los nuevos desafios, Montevideo, CLAEH y UPD-OEA.

R. GENEYRO y M. VÁZQUEZ (2006), "La ampliación de la agenda política y social para el Mercosur actual”, Revista sobre fronteras e integración, Año 11, N²0, Aldea Mundo.

A. MALAMUD (2001), "Democracias presidencialistas y procesos de integración. Hacia un enfoque comparativo del Mercosur", en J. PINTO (Comp.), Argentina entre dos siglos. La política que viene, Buenos Aires, EUDEBA. 
(2002), "Integración regional en América Latina: teorías e instituciones comparadas", en Revista Argentina de Ciencia Política, No 5/6, Buenos Aires, EUDEBA.

A. MALAMUD y L. DE SOUSA (2005), "Regional Parliaments in Europe and Latin America: Between Empowerment and Irrelevance", presentado en el First Global International Studies Conference (World International Studies Committee - WISC), Bilgi University, Istanbul.

D. PAIKIN (2006), "Legitimidad, soberanía y democracia. Debates abordados en torno al Parlamento del MERCOSUR", ponencia presentada en las XIV Jornadas de Jóvenes Investigadores de AUGM, Buenos Aires.

C. PENA y R. ROZEMBERG (2005), "Una aproximación al desarrollo institucional del Mercosur: sus fortalezas y debilidades", Documento de divulgación, No 31, Buenos Aires, BID - INTAL.

F. PEÑA (2005), "Understanding Mercosur and its future", Jean Monnet/Robert Schuman Paper Series, Vol. 5, $\mathrm{N}^{\mathrm{o}}$ 14, The Jean Monnet Chair, University of Miami (USA).

M. VÁZQUEZ (2002), "Sobre la dimensión parlamentaria de los procesos de integración regional. El Mercosur y la Unión Europea en perspectiva comparada a la luz de los desafíos del Área de Libre Comercio de las Américas", en Revista de Ciencia Política, Nº 5/6, Buenos Aires, EUDEBA.

Recibido: 20/05. Aceptado: 07/07 